\title{
Equilibrating Nanoparticle Monolayers Using Wetting Films
}

\author{
Diego Pontoni, ${ }^{1, *}$ Kyle J. Alvine, ${ }^{1, \dagger}$ Antonio Checco, ${ }^{2}$ Oleg Gang, ${ }^{2,3}$ Benjamin M. Ocko, ${ }^{2}$ and Peter S. Pershan ${ }^{1}$ \\ ${ }^{1}$ Department of Physics and SEAS, Harvard University, Cambridge, Massachusetts 02138, USA \\ ${ }^{2}$ Department of Condensed Matter Physics and Materials Science, Brookhaven National Laboratory, Upton, New York 11973, USA \\ ${ }^{3}$ Center for Functional Nanomaterials, Brookhaven National Laboratory, Upton, New York 11973, USA
}

(Received 25 April 2008; published 5 January 2009)

\begin{abstract}
Monolayers of bimodal gold nanoparticles on silicon are investigated by a combination of microscopy (dry monolayers) and x-ray diffraction (dry and wet monolayers). In the presence of an excess of small particles, the nanoscale packing structure closely resembles the small-particle-rich scenario of the structural crossover transition that has been predicted and also observed with micron-scale hard-sphere colloids. Structural morphology is monitored in situ during monolayer dissolution and reassembly within the thin liquid wetting film. This approach allows investigation of size and solvent effects on nanoparticles in quasi-two-dimensional confinement.
\end{abstract}

DOI: 10.1103/PhysRevLett.102.016101

PACS numbers: 68.08.Bc, 61.46.Df, 64.75.Yz

The ability to assemble ordered arrays of nanoscale objects in a reproducible way may lead to functional or smart nanomaterials $[1,2]$. One approach to self-assembly is through controlled evaporation of liquids [3-5] during which the combination of entropic effects associated with the decreasing free volume can combine with the van der Waals attraction, steric repulsion, or electrostatic interactions to produce varying degrees of order [6-8]. Furthermore, the evaporation rate will greatly influence whether the final products are dominated by equilibrium [9] or nonequilibrium [10,11] effects. In this Letter, we show that controlled undersaturated solvent vapors can be used to obtain nanometer thick wetting films that provide conditions of thermodynamic equilibrium during the assembly of nanoparticle arrays. With this approach, preformed dry nanoparticle monolayers are dissolved and reassembled in situ within the thin solvent films. In addition, the liquid film thickness can be regulated to match the different particle sizes involved in the system.

Variations in the nanoparticle size distribution can give rise to different structural motifs $[3,7,12]$. In the case of binary mixtures, size selective phase separation and the formation of ordered binary superlattices are two of the self-assembly phenomena that are expected to take place upon solvent evaporation [7]. The equilibrium phase behavior of binary hard-sphere systems has been studied using density functional theories and numerical simulations $[13,14]$. These studies showed that as the fraction, $\phi$, of small particles is increased, an abrupt change of the system's correlation function is observed over a narrow $\phi$ range. As a consequence, the scattering cross section changes from a form that is dominated by the large particles to one corresponding to the small particles. In the present study we have investigated the case of monolayers obtained using polydisperse materials that are richer in small nanoparticles. The resulting structures are remarkably similar to those recently observed in micron sized model hard-sphere polymer colloids [15], despite (a) the much shorter length scales involved, (b) the different sample preparation method and nature of the particles used, and (c) their large polydispersity. The present measurements of wetting-induced restructuring address the possibility that the similarity to the micron scale results might be a consequence of either the peculiar coating of the nanoparticles [16] or nonequilibrium effects due to fast solvent evaporation during system preassembly. We find that after solvent annealing the small particles still dominate the system's correlation function. In addition, we obtain evidence of enhanced size segregation and size-dependent preferential particle diffusion. The use of thin wetting films opens the way to fundamental studies of size and solvent
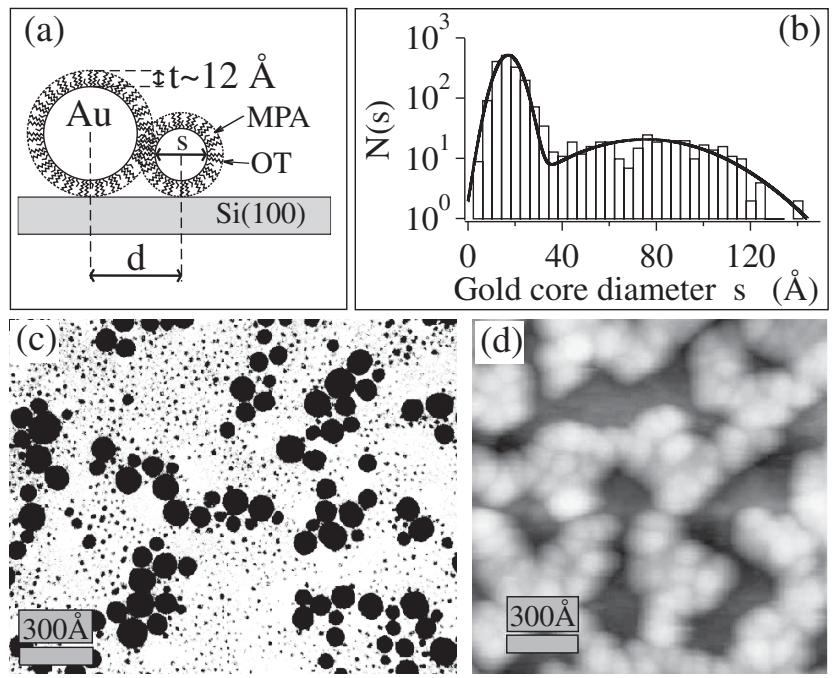

FIG. 1. (a) Illustration of the nanoparticles used. (b) Gold core size distribution histogram from TEM with double Gaussian fit (line). (c) Bright field TEM (contrast enhanced) and (d) AFM images of preannealing nanoparticle monolayers (scale bars $300 \AA$ A). 
effects on the structure and dynamics of nanoparticle assemblies where the confinement can be continuously varied between pure two dimensional (2D) to nearly 3D.

We used thiol-stabilized gold nanocrystals prepared in a solution containing a 2:1 molar ratio of octane-thiol (OT) and mercaptopropionic acid (MPA) [Fig. 1(a)] [16]. The coating shell thickness $(t)$ is dominated by the longer OT chain $(\sim 12 \AA)$. The core size distribution [Fig. 1(b)] obtained from transmission electron microscopy (TEM) images is bimodal with Gaussian mean values $(s)$, standard deviations $(\sigma)$, and polydispersities $(p \equiv \sigma / s)$, respectively, of $s_{s}=17 \AA, \sigma_{s}=5 \AA$, and $p_{s}=29 \%$ for the small particles, and $s_{b}=76 \AA, \sigma_{b}=28 \AA$, and $p_{b}=$ $37 \%$ for the large ones, yielding an average size ratio of $\left(s_{s}+2 t\right) /\left(s_{b}+2 t\right) \simeq 0.4$. Approximately $85 \%$ of the particles are small. They are hydrophobic and disperse readily in toluene. Particle monolayer samples were prepared using the Langmuir-Schaefer approach [17] in which an optimally compressed Langmuir monolayer of particles is transferred by horizontal lift-off from the surface of water to a hydrogen passivated silicon substrate (wafer stamping). Figure 1(c) shows a representative TEM image of a portion of monolayer collected from the water surface onto a standard carbon coated TEM grid. It exhibits correlated regions of the more abundant small particles surrounded by ribbonlike structures of larger particles. The average small-small center-to-center distance is $d_{s}=$ $38 \AA$, whereas the corresponding distance for the big particles is $d_{b}=86 \AA$. Atomic force microscopy (AFM) images of stamped substrates [Fig. 1(d)] highlight the largeparticle structures, while some aspects of the small-particle real-space correlations are confirmed by $x$-ray scattering (to be discussed later, Figs. 2 and 3). Scanning electron microscopy (SEM) images [Fig. 4(a) inset] show that the lift-off method produces cracks (dark regions) in the monolayer (light regions). The resulting macroscopic substrate coverage is approximately $C_{m}=80 \%$. Cracks provide free space allowing monolayer expansion throughout the process of solvent annealing (Figs. 3 and 4).

Toluene vapor is used for the controlled wetting of the nanoparticle monolayers. This is achieved through precise control of the toluene's chemical potential $\mu[18,19]$. For a flat substrate, without a nanoparticle monolayer, a thin liquid film is stabilized by the attractive van der Waals interactions with the substrate, and this gives rise to a wetting film of thickness $\tau \propto \Delta \mu^{-1 / 3}$ [18]. The chemical potential offset from the liquid/vapor coexistence, $\Delta \mu$, is controlled by $\Delta T=T_{s}-T_{r}$, the temperature difference between the substrate $\left(T_{s}\right)$ and the solvent reservoir $\left(T_{r}\right)$. Here $\Delta \mu \approx H_{\text {vap }} \Delta T / T_{r}$, where $H_{\text {vap }}=38.06 \mathrm{~kJ} / \mathrm{mol}$ is the heat of vaporization of toluene [20]. The substrate coated with the dry nanoparticle monolayer is suspended within a chamber [Fig. 2(a)] that is hermetically sealed under nitrogen atmosphere in order to exclude possible water condensation from ambient air. The wetting solvent (tolu-

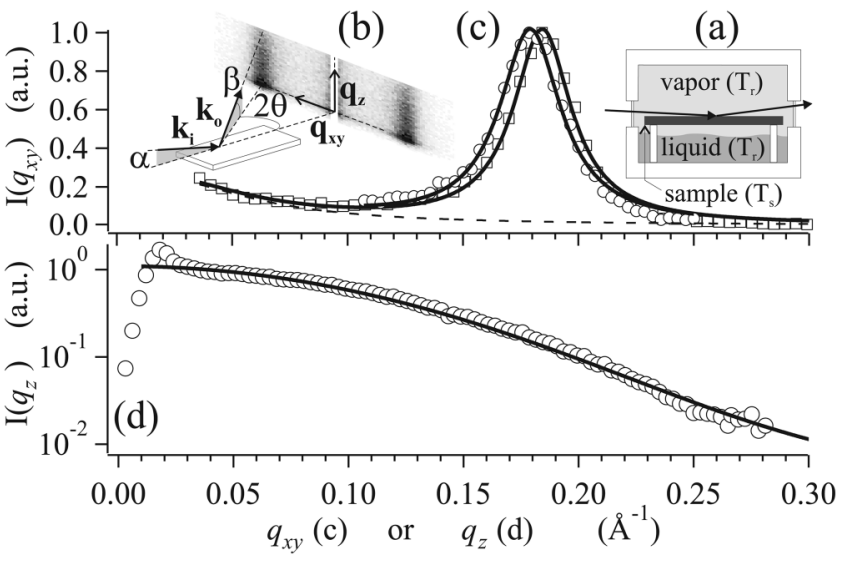

FIG. 2. (a) Illustration of the wetting chamber. (b) Scattering geometry. (c) GID intensity vs $q_{x y}$ with fits (solid lines) for two dry samples. (d) $q_{z}$ scan of the Bragg rod at $q_{x y}=0.18 \AA^{-1}$ and the corresponding fitted polydisperse core-shell form factor for the small particles (solid line).

ene) is injected at the bottom of the cell at temperatures $T_{s}=45^{\circ} \mathrm{C}$ and $T_{r}=30^{\circ} \mathrm{C}$ corresponding to an initial $\Delta T=15 \mathrm{~K}$. The steady state solvent condensation is then reached by stepwise cooling the sample to $\Delta T \simeq 15 \mathrm{mK}$. On flat, bare substrates the wetting liquid thickness increases from $\sim 1 \mathrm{~nm}$ to $\sim 10 \mathrm{~nm}$ for a corresponding 3 orders of magnitude decrease in $\Delta T$ [18]. Solvent is subsequently desorbed by stepwise heating the sample back to $T_{s}=45^{\circ} \mathrm{C}$. The resulting evolution of the nanoparticle equilibrium microstructures is monitored in situ using grazing incidence $x$-ray diffraction (GID). In the present study we applied slow temperature changes (1-

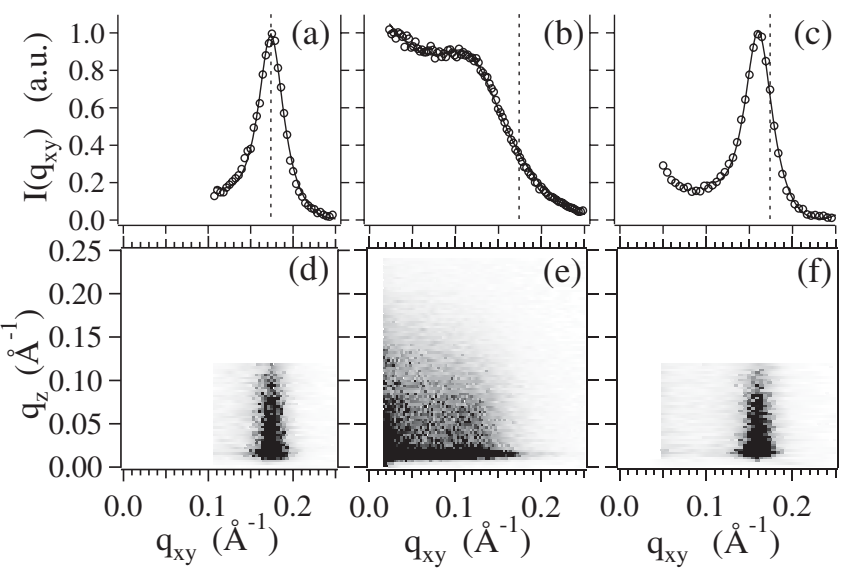

FIG. 3. In situ GID results obtained during controlled wetting with toluene. GID profiles (top) and corresponding 2D scattering patterns (bottom) for the initial thinnest wetting liquid film at $\Delta T=15 \mathrm{~K}(\mathrm{a}, \mathrm{d})$, intermediate thick liquid at $\Delta T=15 \mathrm{mK}$ (b, e), and final thin liquid at $\Delta T=15 \mathrm{~K}(\mathrm{c}, \mathrm{f})$. Continuous lines are Lorentzian fits. Dashed vertical lines indicate the GID peak position at $q_{x y}=0.174 \AA^{-1}$ pertaining to the initial thin liquid film at $\Delta T=15 \mathrm{~K}$. Intensities are independently scaled to one. 
3 degrees/hour) that were separated by long stabilization periods (30-60 minutes).

$\mathrm{X}$-ray measurements were performed at a wavelength of $\lambda=1.535 \AA$ using the Harvard/BNL liquid surface spectrometer [21] at the X22B beam line of the National Synchrotron Light Source. The GID geometry is illustrated in Fig. 2(b), along with a typical diffraction pattern showing the "Bragg rods" characteristic of a 2D arrangement of the particles within a single layer. The x-ray momentum transfer has components normal and parallel to the reflecting surface $\mathbf{q}_{\mathbf{z}}$ and $\mathbf{q}_{\mathbf{x y}}$, where $q_{z}=2 \pi[\sin (\alpha)+$ $\sin (\beta)] / \lambda$ and, at small $\alpha=0.18^{\circ}, q_{x y} \approx(2 \pi / \lambda) \times$ $\left[1+\cos ^{2} \beta-2 \cos \beta \cos 2 \theta\right]^{1 / 2}$. The $2 \mathrm{D}$ scattering patterns were acquired by $2 \theta$ scans with a vertically oriented linear position sensitive detector with slits defining a horizontal resolution of $\delta q_{x y} \simeq 0.01 \AA^{-1}$. Integration of the 2D patterns over the $q_{z}$ range $0-0.12 \AA^{-1}$ yields the 1D profiles presented in Fig. 2(c). Peaks are reasonably well described by the sum (solid line) of a Lorentzian term, which describes the peak at finite $q_{x y}$, and a background term (dashed line) which is proportional to $\left|q_{x y}\right|^{-2}$. The same integration and peak fitting is applied to the diffraction data acquired across the solvent annealing cycle (Fig. 3).

The small differences between the two independent data sets in Fig. 2(c) are representative of sample variations. For the dry sample that was subsequently subjected to toluene annealing (Fig. 3), the single diffraction in-plane peak at $q_{x y}=0.179 \AA^{-1}$ indicates local hexagonal packing with a nearest-neighbor spacing of $d_{0}=4 \pi /(\sqrt{3} \times$ $\left.0.179 \AA^{-1}\right)=40.5 \pm 0.5 \AA$, comparable with the TEM small-particle distance $d_{s}$. In contrast, the peak that would be expected at $\simeq 0.08 \AA^{-1}$ for close packed big particles is not observed. The peak width $\Delta q_{x y} \simeq 0.030 \AA^{-1}$ corresponds to correlated domains of $\xi=2 /\left[\Delta q_{x y}-\delta q_{x y}\right] \simeq$ $100 \AA$. The absence of higher order peaks from the small nanoparticles (data not shown) originates from both the short range of the interparticle correlations and the nanoparticle form factor. The Bragg rod [Fig. 2(d)], $q_{z}$ scan at constant $q_{x y}$, is a measure of the form factor of the particles that contribute to the GID peak. A model (continuous line) based on polydisperse core-shell spheres having structural parameters characteristic of the small particles $\left(s_{s}=17 \AA\right.$, $\sigma_{s}=5 \AA, t=11.5 \AA$ and an electron density ratio of 10 between core and shell) is consistent with the rod data.

After the solvent was introduced at $\Delta T=15 \mathrm{~K}$, the lattice constant increased slightly to $d_{1}=41.7 \pm 0.5 \AA$ [Fig. 3(a)]. At high $\Delta T$ the liquid film is thin, and the scattering pattern still exhibits the typical monolayer Bragg rod [Fig. 3(d)]. During the stepwise cooling (not shown) the GID peak shifted to lower $q_{x y}$, indicating an increase of lattice constant, while an additional contribution of isotropic scattering gradually appeared. At the lowest temperature, $\Delta T=15 \mathrm{mK}$, corresponding to the thickest liquid film, the scattering pattern was nearly radially symmetrical [Fig. 3(e)], with a residual Bragg rod peak centered at $q_{x y} \simeq 0.12 \AA^{-1}$ [Fig. 3(b)] corresponding to $d_{2} \sim 60 \pm 5 \AA$. Subsequent stepwise heating to $\Delta T=$ $15 \mathrm{~K}$ gave rise to an increasing Bragg rod component, decreasing isotropic scattering, and gradual shift of the GID peak back to larger $q_{x y}$ values. At $\Delta T=15 \mathrm{~K}$ [Fig. 3(c) and 3(f)], where the wetting liquid is again thin, the pattern resembles those in Figs. 3(a) and 3(d), albeit with $d_{3}=45.1 \pm 0.5 \AA$.

SEM images [Fig. 4(a)] acquired after completion of the wetting cycle exhibit dark gray regions that are filled mostly with small particles, thus suggesting two wettinginduced structural effects: (i) preferential diffusion of small particles into the initially empty cracks, and (ii) enhanced size segregation within the originally dry monolayer. This is also apparent in AFM images such as Fig. 4(b). Here the large particles are much more abundant within the right portion of the figure (brightness increases with height). Bright spots in the left portion are isolated large particles within a small-particle-rich region, while black spots are holes exposing the underlying substrate. The structures formed by small particles are less compact, and consequently voids are statistically more likely. Figures 4(c)-4(f) schematically illustrate the particle configurations that are consistent with these observations.

The large lattice expansion which occurs in the case of the thick liquid film indicates an increase in area per

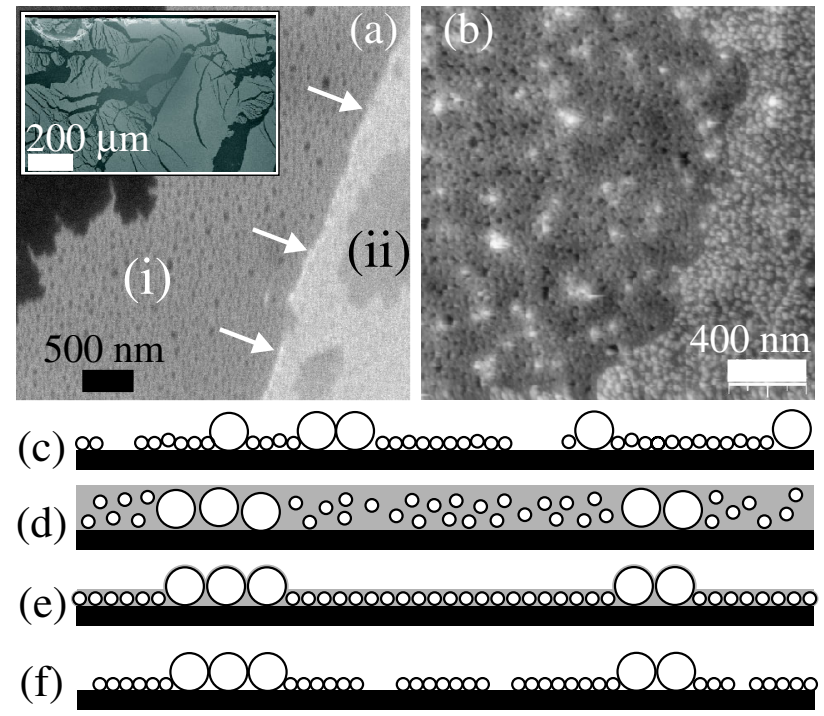

FIG. 4 (color online). Top: SEM (a) and AFM (b) of samples redried after wetting and X-ray experiments. Inset in (a) shows SEM image of a dry sample before solvent annealing; note the cracks (dark) in the monolayer (bright). Arrows in (a) indicate the edge of a crack in the original monolayer. (i) and (ii) indicate small-particle-rich regions (see text). Bottom: pictorial summary of various system conditions studied. (c) Initial dry sample, (d) thickest wetting liquid, (e) annealed sample in thin liquid, (f) redried sample after annealing. 
particle by a factor of $\left(d_{2} / d_{0}\right)^{2}=2.2$. Since there is insufficient unoccupied substrate area for this degree of lateral expansion, some of the particles must move vertically. The isotropic scattering component [Fig. 3(e)] is a signature of this incipient 3D dissolution of the small particles [Fig. 4(d)]. Within $\mathrm{a} \simeq 10 \mathrm{~nm}$ thick solvent film, the small particles experience a nearly $3 \mathrm{D}$ environment, while the large ones are still basically confined to a $2 \mathrm{D}$ geometry. This confinement imbalance may facilitate the preferential diffusion of small particles into void regions, which is also favored by the fact that smaller particles have a larger self-diffusion coefficient. The relative substrate area occupied by small particles in the reassembled monolayer [Figs. 3(c) and 3(f)] has increased by a factor $f=\left(d_{3} / d_{0}\right)^{2}=1.24$. Application of this factor to the entire system (small and large particles) indicates that the macroscopic substrate coverage reached $f C_{m} \simeq$ $100 \%$ [Fig. 4(e)]. This suggests that the particles, still wet by a thin solvent layer, are mobile and explore by $2 \mathrm{D}$ diffusion the entire area available. Coverage in the redried sample [Figs. 4(a) and 4(f)] is again smaller than $100 \%$ as a consequence of complete solvent evaporation.

Solvents play an important role in the assembly of nanoparticle thin films. For the films studied here, toluene adsorption occurs both within the aliphatic chains, within the interstices between neighboring particles, and between the substrate and the first layer of particles. This liquid adsorption appears to reduce the attractive interparticle dispersive interactions, thus resulting in a lattice expansion, consistent with the GID observations. Upon drying, the recovery of the monolayer without the formation of $3 \mathrm{D}$ particle aggregates indicates that the observed wet $2 \mathrm{D}$ films are in thermodynamic equilibrium. Capillary bridging between neighboring particles has been suggested to play an important role in the assembly $[22,23]$. If this was important it would give rise to an additional attractive interparticle interaction that would reduce the interparticle spacing after a small amount of liquid was adsorbed from the dried state. Rather, we observe a slight expansion after a small amount of liquid is introduced to the dry state, and this indicates that attractive capillary bridging plays a minor role compared to adsorption in the interstices. The reassembled monolayer still lacks a GID peak associated with the large particles, thus preserving the analogy with the small-particle-rich scenario of the structural crossover transition.

We have demonstrated that controlled wetting of particle assemblies can be used to explore the delicate nanoscale interplay between particle size and solvent thickness, and its effects on diffusion, phase separation, and order formation in 2D and quasi-3D environments. The approach presented here may enable a host of fundamental studies of the structure and dynamics of nanoconfined particles in solution. Furthermore, wetting-induced annealing of largescale nanoparticle arrays may eventually lead to technological applications.

We thank Francesco Stellacci for providing the particles, Masafumi Fukuto for suggestions on trough operation, and David Bell and Yuan Lu for assistance at the Harvard Center for Nanoscale Systems. Work was supported by the National Science Foundation Grant No. 03-03916. Work at BNL is supported by DE-AC02-76CH0016 through the Division of Materials Science.

* Present address: European Synchrotron Radiation Facility, 38043 Grenoble, France.

pontoni@esrf.fr

†Present address: Pacific Northwest National Laboratory, Richland, WA 99354, USA.

[1] G. M. Whitesides, Small 1, 172 (2005).

[2] I. W. Hamley, Angew. Chem., Int. Ed. 42, 1692 (2003).

[3] P. C. Ohara, D. V. Leff, J. R. Heath, and W. M. Gelbart, Phys. Rev. Lett. 75, 3466 (1995).

[4] B. A. Korgel and D. Fitzmaurice, Phys. Rev. Lett. 80, 3531 (1998).

[5] S. Narayanan, J. Wang, and X. M. Lin, Phys. Rev. Lett. 93, 135503 (2004).

[6] C. B. Murray, C. R. Kagan, and M. G. Bawendi, Science 270, 1335 (1995).

[7] C. J. Kiely et al., Nature (London) 396, 444 (1998).

[8] E. V. Shevchenko et al., Nature (London) 439, 55 (2006).

[9] F. X. Redl et al., Nature (London) 423, 968 (2003).

[10] E. Rabani et al., Nature (London) 426, 271 (2003).

[11] T. P. Bigioni et al., Nature Mater. 5, 265 (2006).

[12] P. Bartlett, R. H. Ottewill, and P. N. Pusey, Phys. Rev. Lett. 68, 3801 (1992).

[13] C. Grodon et al., J. Chem. Phys. 121, 7869 (2004).

[14] C. Grodon et al., Mol. Phys. 103, 3009 (2005).

[15] J. Baumgartl et al., Phys. Rev. Lett. 98, 198303 (2007).

[16] A. M. Jackson, J. W. Myerson, and F. Stellacci, Nature Mater. 3, 330 (2004).

[17] I. Langmuir and V. J. Schaefer, J. Am. Chem. Soc. 59, 2400 (1937).

[18] I. M. Tidswell et al., Phys. Rev. B 44, 10869 (1991).

[19] K. J. Alvine et al., Phys. Rev. B 73, 125412 (2006).

[20] V. Majer and V. Svoboda, Enthalpies of Vaporization of Organic Compounds: A Critical Review and Data Compilation (Blackwell Scientific Publications, Oxford, UK, 1985), p. 300.

[21] D. K. Schwartz, M. L. Schlossman, and P.S. Pershan, J. Chem. Phys. 96, 2356 (1992).

[22] N. D. Denkov et al., Langmuir 8, 3183 (1992).

[23] H. Celio, E. Barton, and K. J. Stevenson, Langmuir 22, 11426 (2006). 